\title{
Enhanced differentiation of isomeric RNA modifications by reducing the size of ions in ion mobility mass spectrometric measurements
}

\author{
Hongzhou Wang ${ }^{1}$, Daniel A. Todd ${ }^{1}$ and Norman H. L. Chiu ${ }^{1,2^{*}}$ (D)
}

\begin{abstract}
With the ability to differentiate different molecular sizes, ion mobility spectrometry (IMS) has great potentials in the analysis of isomeric compounds. However, due to the lack of sensitivity and resolution, IMS has not been commonly used. To address the issue on resolution, the goals of this study are to explore a more effective way to perform IMS by reducing the size of ions prior to the IM measurements, and apply the new approach to the differentiation of isomeric RNA modifications. The size reduction of ribonucleoside ions was effectively accomplished by using the collision-induced dissociation process, in which the N-glycosidic bond in ribonucleoside was cleaved and split the ions into two parts-a smaller nucleobase ion and a neutral molecule of ribose sugar. Since the chemical group that corresponds to most of the RNA modifications makes up a relatively small part of the molecular structure of nucleobases, the differentiation of the dissociated nucleobase ions is expected to require a lower ion mobility resolution than the differentiation of bigger isomeric ribonucleoside ions. By using RNA methylation as a model in this study, the proposed method lowered the required resolution by $16 \%$ for the differentiation of 1-methyladenosine and N6-methyladenosine. Similar results were also obtained from the differentiation of methylated cytidine isomers. In comparison to the results obtained from using the conventional tandem mass spectrometric method, there was no significant loss of signals when the proposed method was used. The proposed method is expected to be applicable to other types of isomeric compounds. Also, the same approach is applicable on other IMS platforms.
\end{abstract}

Keywords: RNA modifications, lon mobility, Size reduction

\section{Introduction}

Ribonucleic acid (RNA) is one of the key components in the living cells and plays many different roles. RNA is sub-divided into several types of RNA, such as message RNA, transfer RNA, and microRNA. In terms of the basic RNA structure, it is made up of only four different building blocks, namely, adenosine, uridine, guanosine, and cytidine. The composition and the order of these

\footnotetext{
* Correspondence: prof.chiu@gmail.com

'Department of Chemistry and Biochemistry, University of North Carolina Greensboro, Greensboro, NC 27412, USA

${ }^{2}$ Joint School of Nanoscience and Nanoengineering, University of North Carolina Greensboro, Greensboro, NC 27401, USA
}

ribonucleotides, i.e., RNA sequence, define the identity of each RNA molecule. However, in order to achieve some of the RNA functionalities, the molecular structure of RNA can be altered by a whole spectrum of posttranscriptional modifications. It is important to note that RNA modifications are different from RNA editing, which focuses on the changes of RNA sequence, whereas RNA modifications refer mainly to the changes in the molecular structure of ribonucleotides. Currently, there are 172 different RNA modifications reported in the literature (Boccaletto 2018). Among them, the most frequent modification is RNA methylation (Zaccara 2019;

\section{Springer Open}

(0) The Author(s). 2020 Open Access This article is licensed under a Creative Commons Attribution 4.0 International License, which permits use, sharing, adaptation, distribution and reproduction in any medium or format, as long as you give appropriate credit to the original author(s) and the source, provide a link to the Creative Commons licence, and indicate if changes were made. The images or other third party material in this article are included in the article's Creative Commons licence, unless indicated otherwise in a credit line to the material. If material is not included in the article's Creative Commons licence and your intended use is not permitted by statutory regulation or exceeds the permitted use, you will need to obtain permission directly from the copyright holder. To view a copy of this licence, visit http://creativecommons.org/licenses/by/4.0/. 
Shi 2019). In the case of monomethylation, the ribonucleotide is modified by the addition of a single methyl group, and the location of the methyl group can be added at different positions. As a result, a group of different isomers are generated. The molecular mass of each isomeric RNA modification is of course identical to each other, but their corresponding physical and chemical properties are slightly different. For instance, two of the isomers of methylated adenosine, namely, 1methyladenosine (m1A) and N6-methyladenosine (m6A), can disrupt their base pairing with uridine, but the extent of disruption is different. Since the proper base pairing between two complementary RNA sequences is crucial to the formation of specific RNA folding or RNA duplexes, it is therefore important to distinguish which of the two isomers are present in the modified RNA molecules.

With the advances in the annotation of genomes, there are growing interests to tackle the analysis of RNA modifications at the transcriptomic level (Licht 2016; Basanta-Sanchez 2016; Nachtergaele 2018; Pan 2018; Huber 2019; Konno 2019). In the literature, this area of research is referred as epitranscriptomic analysis. The ultimate goal of epitranscriptomic analysis is to determine the identity and the frequency of all the RNA modifications in a specific transcriptome as well as pinpointing their exact locations on each transcript. The current approach for carrying out epitranscriptomic analysis can be divided into several categories. Firstly, the next generation sequencing (NGS) and other sequencing-based methods have the advantages of providing the exact location of RNA modifications while being a quantitative analysis (Evans 2017: Zhang et al., 2019b; Zhang et al., 2019a; Hsu and He, 2019; Hsu et al., 2019; Motorin 2019). However, in general, sequencing methods do not generate the detection signals directly from the modified ribonucleotides, thus sequencing methods are prone to have some errors (Grozhik 2018). In many cases, the rate of errors is relatively low and acceptable. The major drawback of sequencing methods is the incapability to detect all the known RNA modifications in a single experiment. The same drawback applies with the probebased methods. An alternative approach is a technology that measures the variation of current going across a nanopore when a strand of nucleic acid is passing through the nanopore (Kono 2019). In principle, the nanopore technology can perform the RNA sequencing at relative high speed. However, its applicability for the detection of RNA modifications is not fully demonstrated yet. Therefore, until a more suitable method will become available for analyzing the epitranscriptome, the conventional approach of using liquid chromatography mass spectrometry (LC-MS) method to analyze the digested ribonucleosides remains as the most comprehensive approach for profiling all the RNA modifications in a specific epitranscriptome (Basanta-Sanchez 2016; Huber 2019; Wetzel 2016; Manasses 2018). When using the LC-MS method to accurately identify a specific RNA modification, it requires the accurate mass of the modified ribonucleoside ion and at least one of the corresponding CID (collision-induced dissociation) fragment ion to be detectable. To differentiate the isomeric RNA modifications, it also requires a unique CID fragment ion that corresponds to the isomer of interest to be detectable. This in turn may require more precursor ions to be available for the CID process. For LC-MS analysis, the amount of a specific precursor ions is dependent on a number of factors, which include the sample concentration, the flow rate of the mobile phase, the ionization efficiency of the eluted sample, and the duration of the time window during which the sample is being eluted from the LC column. In addition, the use of an optimal CID energy may require to break off the unique CID fragment ion from the precursor ion. Hence, in practice, the signal that corresponds to the unique CID fragment ion is not always detectable. One way to address this issue is to couple ion mobility (IM) spectrometry to the MS measurements. In general, IM spectrometry focuses on measuring the differences in size, shape, and number of charge on the molecular ions (May 2015; Ewing 2016; Gabelica 2018; Kirk 2019). Hence, IM measurements are independent to the MS measurements. From the perspective of sample identification, the IM data can be used to support the MS data and further enhance the accuracy on the sample identification. The Fabris group had published the first chromatographic-free report on using IM-MS to analyze epitranscriptome (Rose 2015). More recently, the Kammerer group had used the same IM-MS method to determine the profile of ribonucleosides that exist in cell culturing medium of different cancer cell lines (Lagies 2019). In both cases, the reported arrival time distribution (ATD) of each individual isomeric ribonucleoside ion were very close to each other and might exceed the available ion mobility resolution (Giles 2011). In this report, by using the same IM platform, our goals are to explore a way to achieve a more effective IM separation by reducing the size of ions prior to the IM measurements and apply the improvement to enhance the accuracy for identifying specific isomeric RNA modifications.

\section{Materials and methods}

Adenosine and N1-methyladenosine (m1A) were obtained from Sigma-Aldrich (St. Louis, MO, USA). N6Methyladenosine (m6A), 3-methylcytidine (m3C), and 5methylcytidine $(\mathrm{m} 5 \mathrm{C})$ were purchased from Carbosynth (Compton, Berkshire, UK). Acetonitrile (ACN), formic acid, and water at the Optima LC/MS grade were 
purchased from Thermo Fisher Scientific (Hampton, $\mathrm{NH}$, USA). All the stock solution (5 $\mathrm{mM})$ of unmodified and modified ribonucleoside were prepared with deionized water, and stored at $-20^{\circ} \mathrm{C}$. In each experiment, $70 \mu \mathrm{M}$ of freshly diluted standard solution was prepared with $50 \% \mathrm{ACN} /$ water and $0.01 \%$ formic acid, which resembles the mobile phase being used in our laboratory for the conventional LC-MS/MS analysis of specific epitranscriptomes.

\section{Ion mobility mass spectrometric measurements}

All experimental data was acquired using the Waters Synapt G2 high definition mass spectrometer (Waters, Milford, MA, USA), which was equipped with an electrospray (ESI) source. Each sample was delivered by direct infusion at a flow rate of $10 \mu \mathrm{L} / \mathrm{min}$. The source temperature was set at $80^{\circ} \mathrm{C}$, the desolvation temperature at $200^{\circ} \mathrm{C}$, the gas flow rate at the cone was maintained at $50 \mathrm{~L} / \mathrm{h}$, and the desolvation gas flow rate at $800 \mathrm{~L} / \mathrm{h}$ while the other ESI parameters were optimized to attain the highest mass spectrometric signal without any detectable in-source fragmentation. Unless otherwise stated, the traveling wave ion mobility (TWIM) components were operated under the default settings, which included a flow of Argon gas to the trap and transfer cell at $2 \mathrm{~mL} / \mathrm{min}$, a flow of helium gas to the helium cell at $180 \mathrm{~mL} / \mathrm{min}$, a flow of nitrogen gas to the ion mobility cell at $30 \mathrm{~mL} / \mathrm{min}$, the wave height at $30 \mathrm{~V}$, and the wave velocity at $1200 \mathrm{~m} / \mathrm{s}$. The time-offlight (TOF) mass analyzer was operated under the resolution mode, and the negative ion mode was used. After switching to the IM mode, approximately $5 \mathrm{~min}$ wait time was given for the pressure within the TWIM components to be stabilized. In each experiment, the signals from each sample were acquired for $30 \mathrm{~s}$. At the end of measuring each standard dilution, the setup for carrying out the direct infusion and the ion source were rinsed out with at least $1 \mathrm{~mL}$ of $50 \% \mathrm{ACN} /$ water at the maximum flow rate $(100 \mu \mathrm{L} / \mathrm{min})$. All the data acquisition and analysis were carried out with the MassLynx software program (version 4.1) from waters.

\section{Results and discussion}

\section{Enhanced ion mobility (IM) separation of isomeric RNA modifications}

Although the presence of structural isomers is well known, the differentiation of different isomers, including those among RNA modifications, remains as a challenging task. The goal of this investigation is to explore a more effective way to differentiate ribonucleoside ions by using IMS, such that IM data can be used to support the conventional tandem mass spectrometric (MS/MS) data and more accurate identification of isomeric RNA modifications can be accomplished. To enhance the IM separation of ribonucleoside ions, our strategy is to reduce the size of ribonucleoside ions. The rationale of this concept stems from the fact that there are only four canonical ribonucleotides in RNA molecules, namely, adenosine, uridine, guanosine, and cytidine. Partly due to the simplicity of RNA structure, modified RNAs are normally digested into single ribonucleotides prior to the measurements. The majority of the known RNA modifications involve the addition of a small chemical group. For instance, adenosine can be monomethylated, and results in 1-methyladenosine $(\mathrm{m} 1 \mathrm{~A})$ or N6methyladenosine (m6A), which are the two most common RNA modifications in eukaryotes. The methyl group makes up only $5 \%$ of the molecular mass of m1A or m6A. Therefore, it has been challenging to distinguish the two isomeric ions by using the current ion mobility techniques. If the size of those methylated ribonucleosides is reduced by cutting off the ribose, the same methyl group would make up $10 \%$ of the remaining molecular mass. Therefore, the proposed size reduction is equivalent to amplifying the influence from the methyl group (or other RNA modifications) on the resulting molecular shape. From the perspective of IMS, the smaller the ions that can be generated through size reduction, the bigger the difference can be generated in the molecular shapes between two isomeric ions. To evaluate this concept, the collision cross section (CCS) of $\mathrm{m} 1 \mathrm{~A}$ and $\mathrm{m} 6 \mathrm{~A}$ with or without removing the ribose was calculated. The results are summarized in Table 1. By cross checking the calculated CCS value of m1A with the experimentally measured CCS values in the unified CCS compendium, the CCS calculations in Table 1 were determined to be accurate. More importantly, the results in Table 1 indicate the removal of ribose from $\mathrm{m} 1 \mathrm{~A}$ and $\mathrm{m} 6 \mathrm{~A}$ ions would theoretically lower the required resolution for differentiating the two selected isomers by $16 \%$ (Table 1). In other words, it becomes easier to resolve the smaller methylated adenine ions than the corresponding larger methylated adenosine ions.

Theoretically, the glycosidic bond between the nucleobase and the ribose sugar within the molecular structure

Table 1 Comparison of collision cross section (CCS) and minimum resolution $(R)$ that is required to resolve the peaks of selected isomeric ions in an ion mobility spectrum ( $R=$ CCS/ $\triangle C C S$ ). The CCS calculations were carried out by using the ion mobility spectrometry suite (IMoS) via the parallelized user interface (ParsIMoS) (Kune 2018)

\begin{tabular}{lll}
\hline Isobaric ions & Calculated CCS $(\AA)$ & Required resolution \\
\hline m1A & 164.1 & 36.67 \\
m6A & 168.7 & \\
m1a & 124.7 & 30.69 \\
m6a & 128.9 & \\
\hline
\end{tabular}

$A$ adenosine, $a$ adenine, $\triangle C C S$ difference between two CCS values 
of ribonucleotides is relatively weak. According to the earlier reports, the dissociation of the $\mathrm{N}$-glycosidic bond did occur frequently under the normal collision-induced dissociation (CID) conditions of MS/MS experiments, and led to a neutral loss of the ribose while the positive charge remained on the nucleobase ions (Supplemental Fig. 1.) (Ham 2016). Since the nucleobase has been the target for majority of the known RNA modifications (Boccaletto 2018), the loss of the ribose does not represent any significant drawback to the proposed sizereduction ion mobility (SRI) method.

In this study, all IM measurements were carried out on a highly flexible IM platform named Waters Synapt G2, which is equipped with the traveling wave ion mobility (TWIM) technology and allows accurate mass spectrometric measurements to be carried out simultaneously (Giles 2011). In order to simplify our study, the selected ribonucleoside standards were directly infused into the instrument via an electrospray ionization source. Depending upon the actual sample complexity, the deviation from the standard approach of using liquid chromatography to separate the ribonucleosides prior to MS or IM-MS measurements is theoretically feasible and has been reported (Rose 2015). As indicated in Fig. 1, prior to the IM separation, ions with specific mass-tocharge ratios $(\mathrm{m} / \mathrm{z})$ are selected by a quadrupole mass filter. In order to convert the continuous flow of ions exiting from the quadrupole into batches of ions for TWIM, a trap cell is inserted in between the quadrupole and the IM cell (Fig. 1). At the rear end of the IM cell, there is also a transfer cell. By increasing the voltage at the entrance of the trap or transfer cell, the ion energy can be increased, and leads to the collision-induced dissociation of ions. Under the default operations for IM measurements, the CID of ions is normally set up to occur in the transfer cell after the IM separation is completed (Fig. 1). In order to maximize the yield of different CID fragment ions, there is an option to carry out CID in both trap and transfer cells. For structural analysis, the dual CID approach is referred as time aligned parallel (TAP) fragmentation (Damen 2008). In the TAP analysis, the resulting CID fragment ions in the trap and transfer cells are aligned through their arrival time distribution (ATD). The experimental approach of our proposed method is similar to the TAP analysis, in which the selected ions are dissociated in both trap and transfer cells. In contrast to the TAP analysis, our proposed method aims to reduce the size of the precursor ions by carrying out a very limited ion dissociation in the trap cell; thus, the minimum amount of CID energy is used to avoid the generation of multiple CID fragment ions. In addition, our proposed method does not require any time aligned parallel fragmentation to identify the isomeric ions. Generally speaking, the approach of reducing the size of isomeric ions in the trap cell is equivalent to a step of processing the sample ions before the IM measurements are carried out.

For the proof of concept of the SRI method, two of the isomeric methylated adenosine $(\mathrm{m} 1 \mathrm{~A}$ and $\mathrm{m} 6 \mathrm{~A})$ are used as our initial model. As shown in Fig. 2a, despite of our initial efforts to optimize the key parameters of

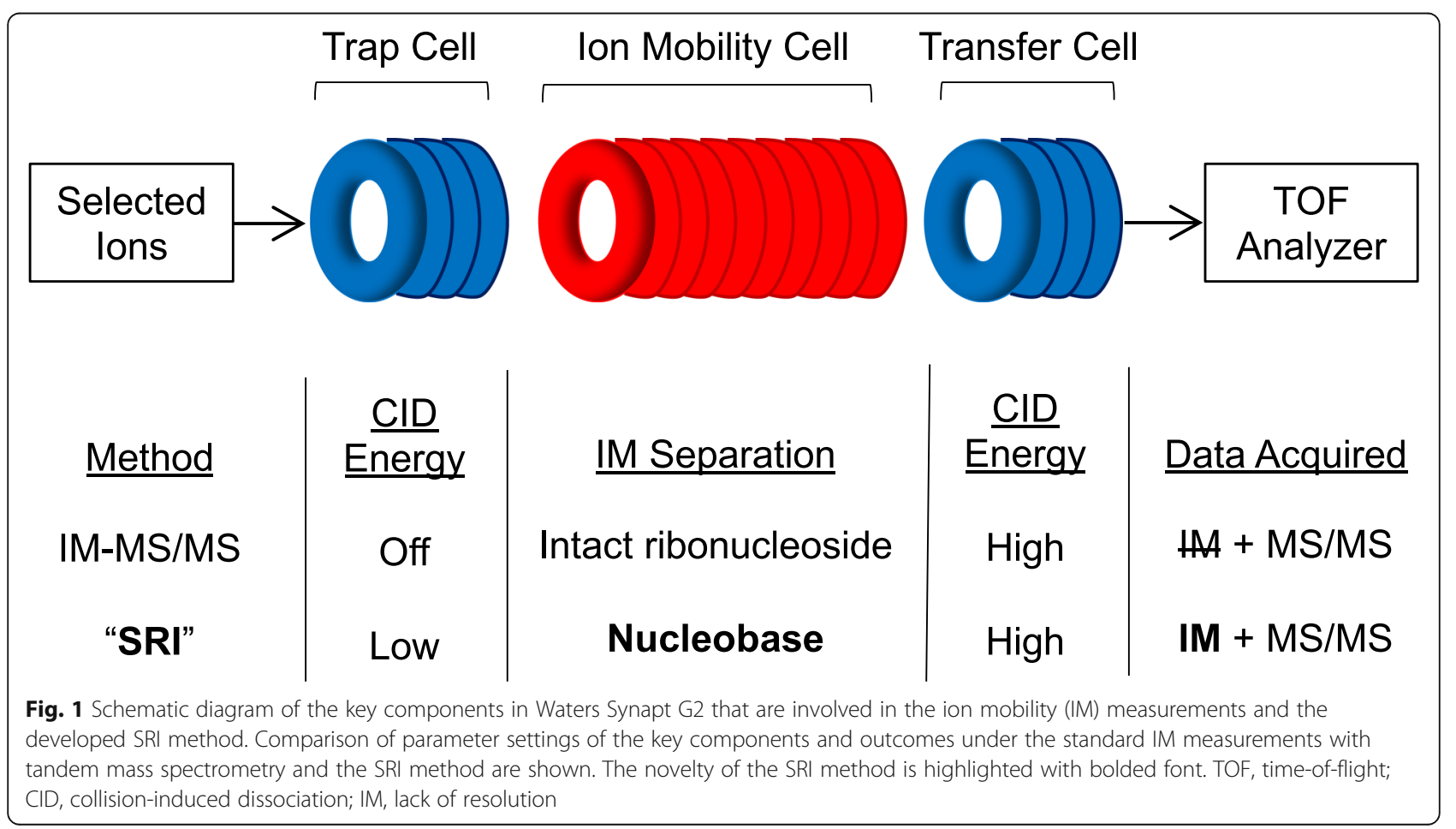


(A)

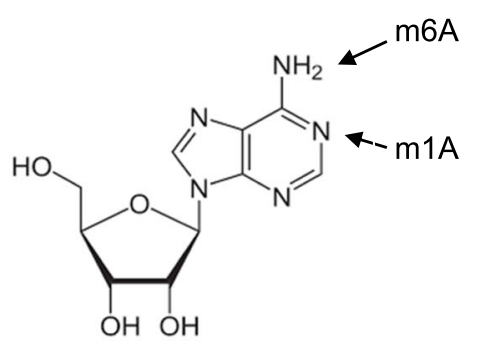

1.61

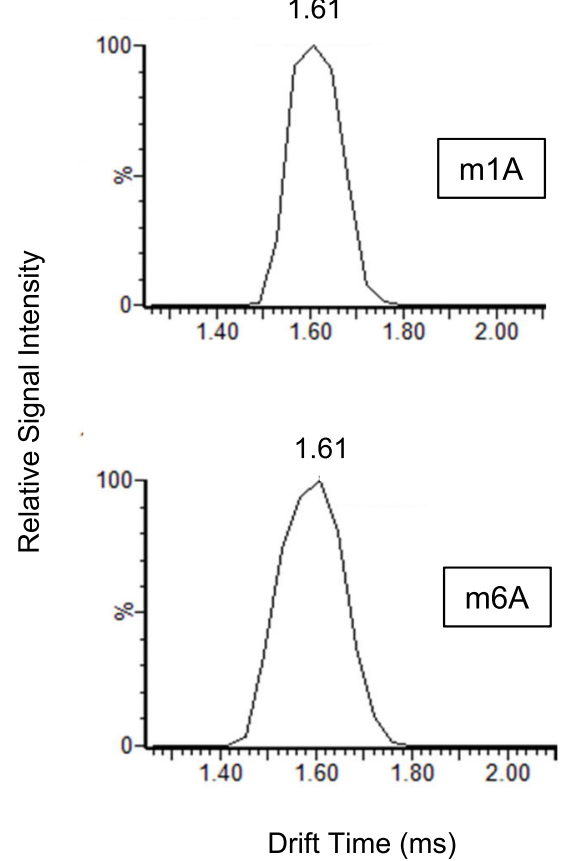

(B)<smiles>Nc1ncnc2[nH]cnc12</smiles>
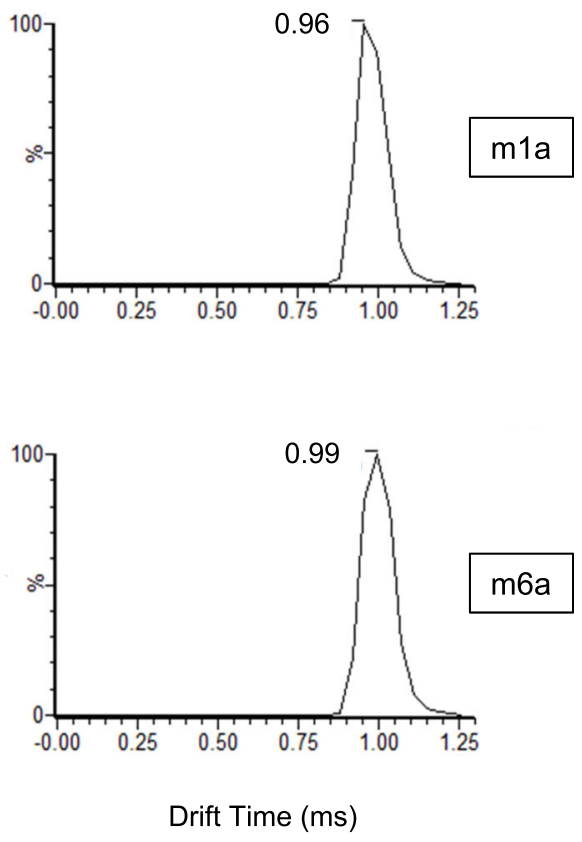

Fig. 2 a Molecular structure of $\mathrm{m} 1 \mathrm{~A}$ and $\mathrm{m} 6 \mathrm{~A}$, and their corresponding extracted ion mobility spectra which were acquired by using the standard IM method. b Molecular structure of mla and m6a, and their corresponding extracted ion mobility spectra which were acquired by using the SRI method. A, adenosine; a, adenine

TWIM, namely, the height and the velocity of traveling waves, the two isomeric methylated adenosine ions remain unresolvable, i.e., equal arrival time distribution (ATD) (Gabelica 2019). Although slightly higher ion mobility resolution was reported for measuring isomeric RNA modifications (Lagies 2019), it does not hinder the development of the proposed SRI method. As shown below, the results in Fig. 2a simply serve as a reference in this study. To set up the SRI method, optimal CID energy in the trap cell is required. The results indicated that the default setting at $4 \mathrm{~V}$ of CID energy was sufficient to achieve a complete dissociation of the $\mathrm{N}$ glycosidic bond in both selected methylated ribonucleoside ions. In Fig. 2b, by using the SRI method, the ATD of the two smaller methylated adenine ions (m1a vs $\mathrm{m} 6 \mathrm{a}$ ) are distinguishable from each other when the same TWIM parameter settings as in Fig. 2a were used. Based on the difference between ATD, the N6-methyladenine (m6a) ion is expected to have a larger CCS than the 1methyladenine (m1a) ion. This observation complies with their calculated CCS values as shown in Table 1 . To ensure the ATD measurement of m6a ion is repeatable, fresh m6A samples were prepared on three different days and the IM measurements were repeated at least three times on each day. The results are highlighted in Fig. 3 and show the SRI method for measuring the ATD of smaller ribonucleoside ions is repeatable.

\section{MS/MS measurements of dissociated nucleobase ions}

As shown above, the ATD measurements could provide a mean to identify the selected isomeric RNA modifications. However, in order to achieve a higher accuracy, the post-IM MS/MS measurements of the smaller ions are needed. By default, the trap cell is the designated 


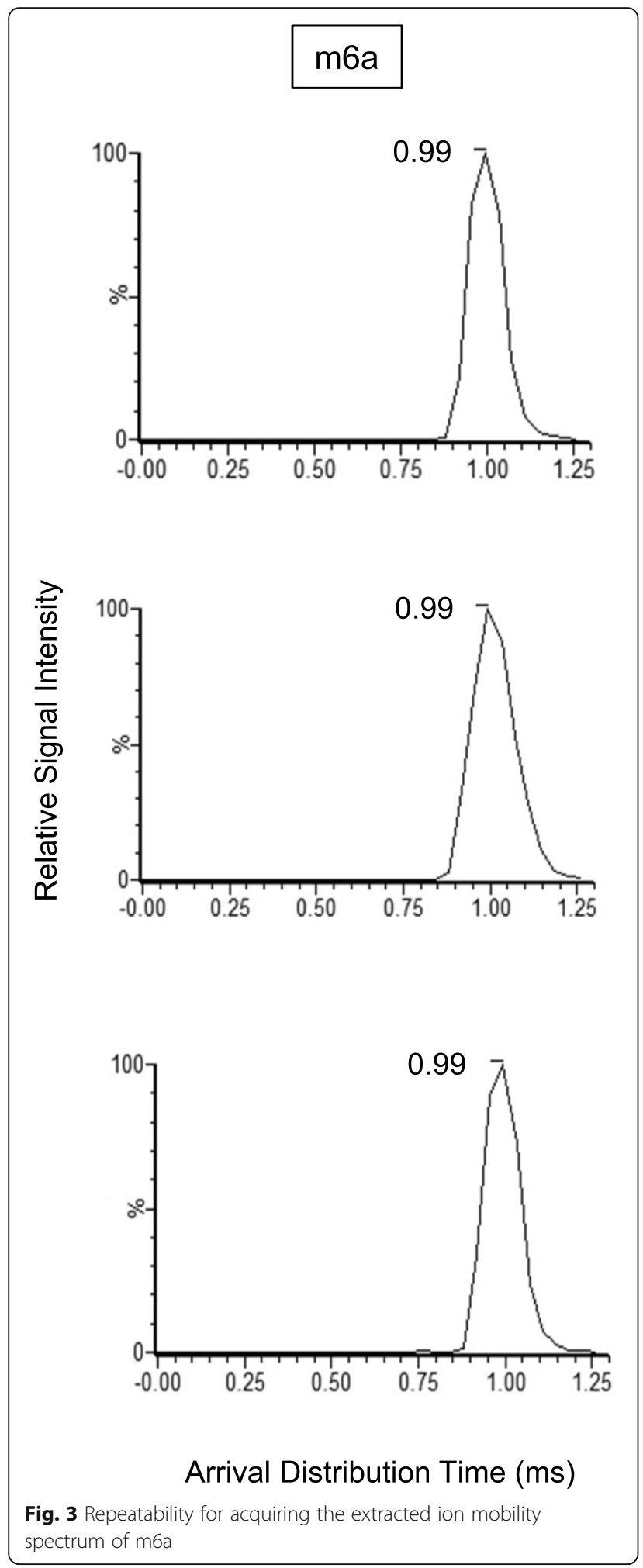

CID cell for MS/MS experiments. However, when using the SRI method, the transfer cell is the only option for carrying out the MS/MS experiments (Fig. 1). Although the transfer cell has the same design and dimension as the trap cell, their parameter settings are different. Furthermore, based on the results in our earlier study, the internal energy of precursor ions was found to be elevated when the stacked rings ion guides were used to transmit ions in the Waters Synapt G2 instrument (Mwangi 2018). Owing to these reasons, the effectiveness of using the transfer cell to carry out the MS/MS experiments was investigated. After optimizing CID energy, the spectral pattern obtained from using the transfer cell to fragment the m6a ions was found to match with the results obtained from using the trap cell to fragment the m6A ions under the standard MS/MS mode (Fig. 4). In the latter case, due to the use of relative high CID energy $(80 \mathrm{~V})$, the precursor ion of m6A was not detectable and only the methylated adenine ion (149.99 $\mathrm{m} / \mathrm{z}$ ) was detected. With similar level of signal intensity in both MS/MS spectra shown in Fig. 4, it represents that there is no compromise on using the SRI method in comparison to the standard MS/MS method. Together with the ATD results in Fig. 2b, it demonstrates the SRI method is a viable approach to distinguish the two selected isomeric RNA modifications with higher accuracy than the standard MS/MS method.

\section{Application of SRI method to other isomeric RNA modifications}

To demonstrate its applicability, the SRI method was also used to detect and distinguish the most common isomeric modification of cytidine, namely, 3methylcytidine (m3C) and 5-methylcytidien (m5C). Through the size reduction of their corresponding precursor ions as described above, a unique and reproducible ATD could be measured for each dissociated cytosine ions ( $\mathrm{m} 3 \mathrm{c}$ vs $\mathrm{m} 5 \mathrm{c}$ ) without any further optimization of the parameter settings. The results are shown in Fig. 5a. In comparison to the results obtained from the analysis of m1a and m6a ion in Fig. $2 b$, there is a slightly bigger difference in ATD between $\mathrm{m} 3 \mathrm{c}$ and $\mathrm{m} 5 \mathrm{c}$ ion. This is attributed to cytosine that has a smaller size than adenine.

To complete the analysis, the MS/MS measurements of $\mathrm{m} 3 \mathrm{c}$ and $\mathrm{m} 5 \mathrm{c}$ ions were carried out. A unique CID fragment ion with $\mathrm{m} / \mathrm{z}$ of 95.01 was easily detectable from the precursor ion of $\mathrm{m} 3 \mathrm{c}$, which could be used to distinguish the two isomeric compounds (Supplemental Fig. 2). As indicated in Fig. 5b, the results obtained from the CID of $\mathrm{m} 3 \mathrm{c}$ ion in the transfer cell under the SRI method is comparable to the results that were obtained from the CID of $\mathrm{m} 3 \mathrm{C}$ ion in the trap cell under the standard MS/MS mode. Thus, the SRI method does support acquiring the same level of structural information as in the case of using the standard MS/MS method while enriching the data set with the ATD of the dissociated nucleobase ions. Overall, the results in Fig. 5 


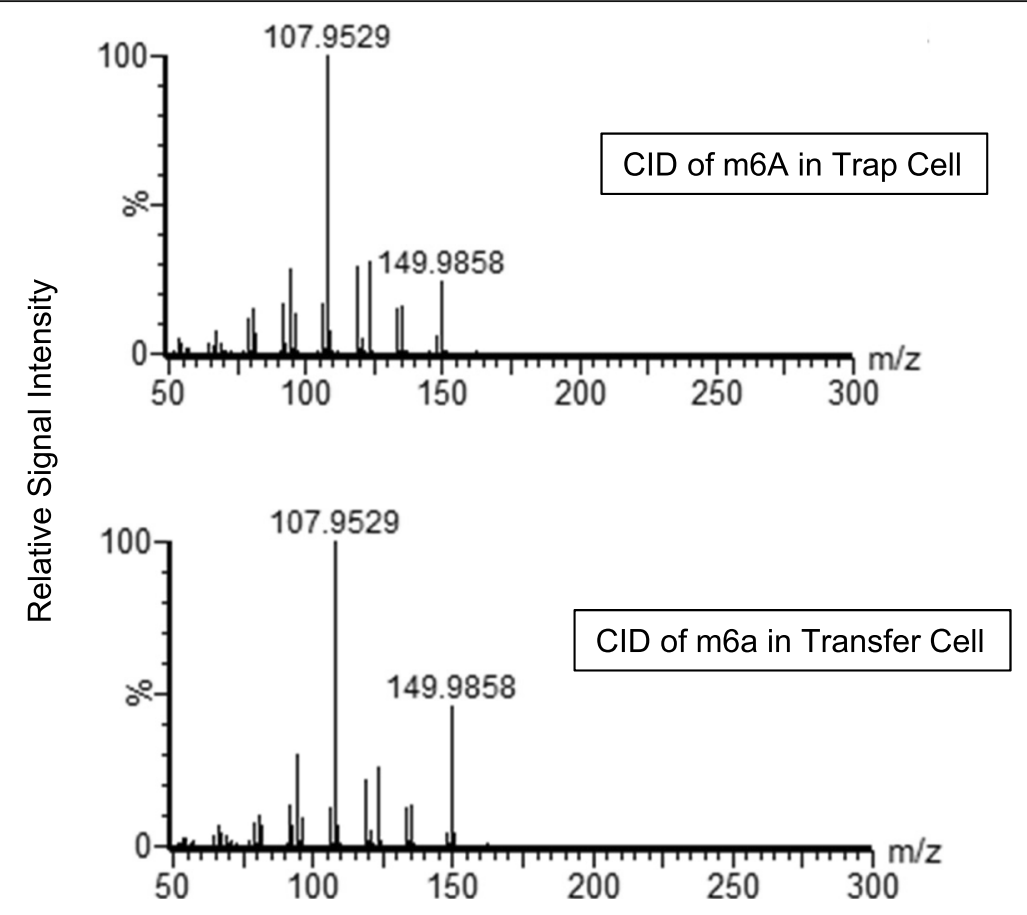

Fig. $4 \mathrm{MS} / \mathrm{MS}$ spectrum of m6A in the trap cell under the standard MS/MS method or m6a in the transfer cell under the SRI method. The CID energy used in the trap and transfer cell was $80 \mathrm{~V}$ and $160 \mathrm{~V}$, respectively. The ion counts for $107.9529 \mathrm{~m} / \mathrm{z}$ ion in both cases reached $2 \mathrm{e}+4$ and $5 \mathrm{e}+4$, respectively

(A)

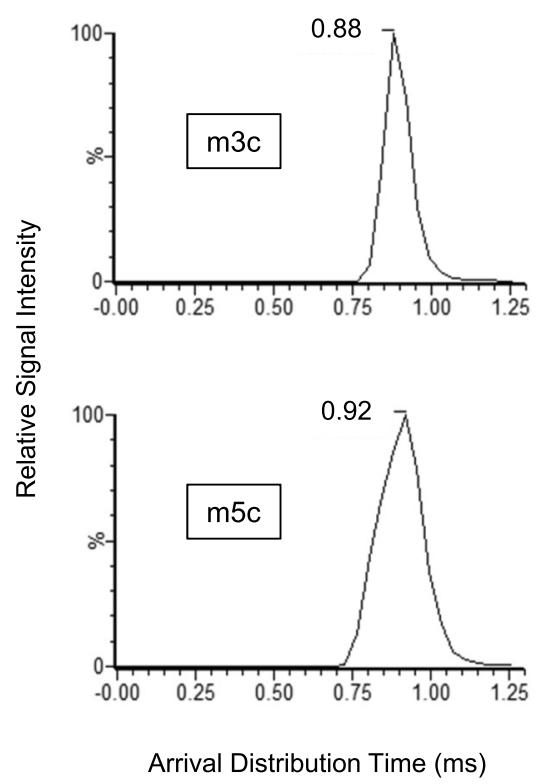

(B)
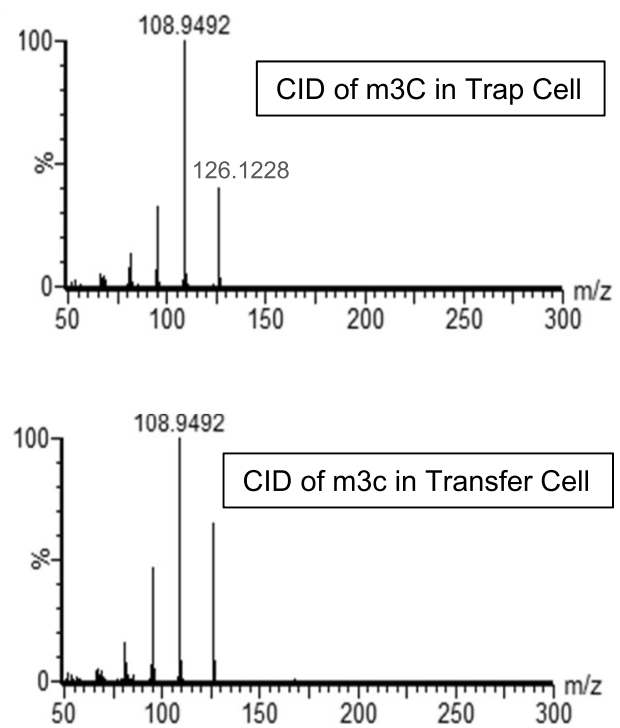

Fig. 5. a Extracted ion mobility spectra of nucleobases that resulted from using the SRI method. $\mathbf{b}$ MS/MS spectrum of m3C in the trap cell under the standard MS/MS method or m3c in the transfer cell under the SRI method. The CID energy used in the trap and transfer cell was $80 \mathrm{~V}$ and $140 \mathrm{~V}$, respectively. The ion counts for $108.9492 \mathrm{~m} / \mathrm{z}$ ion in both cases reached $5 \mathrm{e}+4$ and $4 \mathrm{e}+4$, respectively. m3c, 3-methylcytosine; $\mathrm{m} 5 \mathrm{c}$, 5methylcytosine; m3C, 3-methylcytidine 
demonstrate the SRI method can be used to detect and distinguish other isomeric RNA modifications.

\section{Quantitative measurements using SRI method}

Although quantitative mass spectrometry has already been extended to the analysis of many RNA modifications including RNA methylation (Huber 2019; Wetzel 2016: Manasses 2018), the effectiveness of using the Waters Synapt G2, in which ion mobility spectrometry is coupled to mass spectrometry, for performing quantitative analysis of RNA modifications especially under the SRI method is unknown. In order to ensure the SRI method can be applied to determine the level of specific RNA methylation, the calibration experiments with the standard dilution of $\mathrm{m} 6 \mathrm{~A}$ and $\mathrm{m} 3 \mathrm{C}$ were carried out. Since both ATD and MS/MS measurements are included in the SRI method, the calibration can be carried out in two different ways. The results are shown in Fig. 6. In the case of using m6A as the calibrant, the MS/MS measurements were calibrated with the signal corresponding to the methylated adenine ion at $149.99 \mathrm{~m} / \mathrm{z}$. The reason for choosing this particular CID fragment ion is because there is no unique CID fragment ion that can be used to distinguish the two selected isomers of methylated adenosine. To overcome this issue, Limbach and his associates had used the ratio of signal intensities between methylated adenine ion $(149.99 \mathrm{~m} / \mathrm{z})$ and another CID fragment ion $(107.95 \mathrm{~m} / \mathrm{z})$ that has a higher signal to distinguish the two isomers (Manasses 2018). Since the detection of both signals were required, the MS/MS calibration with m6A was performed by using the lower signal at $149.99 \mathrm{~m} / \mathrm{z}$. In the case of using $\mathrm{m} 3 \mathrm{C}$ as the calibrant, the signal at 95.01 $\mathrm{m} / \mathrm{z}$ that corresponded to a unique CID fragment ion of $\mathrm{m} 3 \mathrm{C}$ was used. For both MS/MS calibrations, the linear dynamic range has about two orders of magnitude and the $R$-squared values are equal or above 0.99 (Fig. 6). The limit of detection of m6A is slightly lower than the detection of $\mathrm{m} 3 \mathrm{C}$. This is mainly because the yield of m6A ion was higher than that of $\mathrm{m} 3 \mathrm{C}$ ion. By comparing the results in Fig. 6 with those obtained by using the standard MS/ MS mode on the same platform, under which the IM operation was turned off and the CID was carried out in the trap cell instead of the transfer cell, no significant difference on both limits of detection was noticed (Supplemental Fig. 3).

For the IM calibration, the signal that corresponds to the dissociated methylated nucleobase in the ion mobility spectrum was used. Based on the design of the SRI method, the IM signal and the MS/MS signals were acquired simultaneously. Therefore, the IM calibration experiments were carried out at the same time with the MS/MS calibration experiments. The results are also shown in Fig. 6. Due to the wider peaks in the ion mobility spectra, the peak area instead of the peak height was used to plot the calibration graphs in Fig. 6. In both cases, the $R$-squared values are slightly below 0.99 . This could be due to the fact that the yield of the dissociated nucleobase ions became lower when the concentration of the parent ribonucleoside ions reached to the ion capacity of the transfer cell. The results in Fig. 6 show that IM calibration is comparable to the conventional MS/ MS calibration. Overall, we show the SRI method can also be used to determine the amount of specific isomeric RNA methylation.

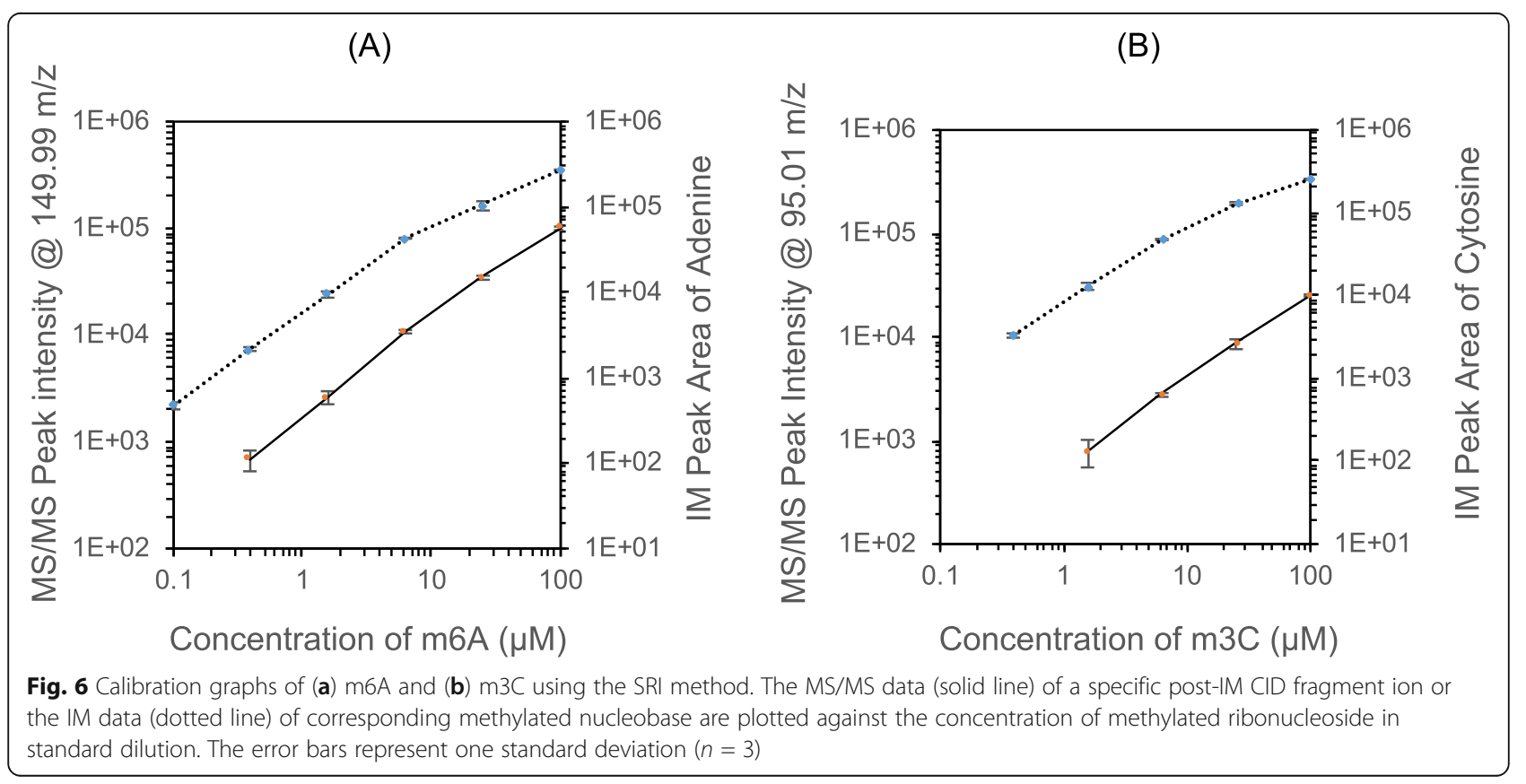




\section{Conclusions}

In general, two of the drawbacks of ion mobility spectrometry are the lack of sensitivity and resolution. The development of the SRI method represents a complementary effort to address the limitation on ion mobility resolution. To the best of our knowledge, this is the first report that uses the concept of size reduction of ions to directly address the limitation on resolution. Equally important, in comparison to the standard MS/MS method, no significant decrease on the sensitivity was found when the SRI method was used. Furthermore, the ability to detect the selected isomers with distinguishable ATD represents an extra dimension of data is available for the identification of isomeric RNA modifications. Another advantage of using the SRI method is the acquisition of ATD is independent to the MS/MS measurements. Specifically, the ATD measurements are dependent on whether the optimal CID energy is being used neither during the MS/MS measurements nor the actual time available to acquire sufficient MS/MS signals. From the perspective of MS/MS measurements, both parameters are often critical and determine whether specific CID fragment ion(s) is detectable or not. The SRI method is expected to be applicable to other types of isomeric compounds. Also, it is technically feasible to adopt the concept of the SRI method on other existing or future IMS platforms, in which different types of ion mobility technology is utilized.

\section{Supplementary information}

Supplementary information accompanies this paper at https://doi.org/10. 1186/s40543-020-00243-5.

Additional file 1: Figure S1. Mechanism for the most frequent collision induced dissociation of ribonucleoside ion.

Additional file 2: Figure S2. MS/MS spectra of $\mathrm{m} 3 \mathrm{c}$ and $\mathrm{m} 5 \mathrm{c}$ in the transfer cell under the SRI method. The unique CID fragment ion at 95.01 $\mathrm{m} / \mathrm{z}$ for $\mathrm{m} 3 \mathrm{c}$ precursor ion is marked with an arrow. The CID energy used in the transfer cell was $80 \mathrm{~V}$.

Additional file 3: Figure S3. Calibration graphs of (A) m6A and (B) $\mathrm{m} 3 \mathrm{C}$ by using the standard MS/MS mode. The CID was taken place in the trap cell with the optimal CID energy. The error bars represent one standard deviation $(n=3)$.

\section{Abbreviations \\ ACN: Acetonitrile; ATD: Arrival time distribution; CID: Collision-induced dissociation; CCS: Collision cross section; ESI: Electrospray ionization; IM: Ion mobility; LC: Liquid chromatography; m1A: 1-Methyladenosine; m6A: N6- Methyladenosine; m1a: 1-Methyladenine; m6c: N6-Methyl adenine; m3C: N3- Methylcytidine; m5C: 5-Methylcytidine; m3c: N3-Methylcytosine; m5c: 5- Methyl cytosine; MS: Mass spectrometry; MS/MS: Tandem mass spectrometry; m/z: Mass-to-charge ratio; SRI: Size reduction ion mobility; TAP: Time-aligned parallel; TOF: Time-of-flight}

\section{Acknowledgements}

The authors wish to thank Joseph Mwangi and Jennifer Simpson for their helpful discussion and the continuous support on the mass spectrometry facilities from the Department of Chemistry and Biochemistry at UNCG.

\section{Authors' contributions}

H.W. performed the experiments, analyzed the data, and wrote the manuscript. D.T. provided the training and supported the IM-MS experiments. N.C. conceived the project, designed the experiments, analyzed the data, and wrote the manuscript. The authors read and approved the final manuscript.

\section{Funding}

H.W. was supported by a teaching assistantship from UNCG. N.C. was a recipient of the Faculty First Award from UNCG.

\section{Availability of data and materials}

Not applicable.

\section{Competing interests}

The authors declared that they have no competing interests.

Received: 7 August 2020 Accepted: 6 October 2020

Published online: 20 October 2020

\section{References}

Basanta-Sanchez M, et al. Attomole quantification and global profile of RNA modifications: epitranscriptome of human neural stem cells. Nucleic Acids Res. 2016;44(3):e26.

Boccaletto P, et al. MODOMICS: A database of RNA modification pathways. 2017 Update. Nucleic Acids Res. 2018;(46):D303-7.

Damen CWN, et al. Electrospray ionization quadrupole ion-mobility time-of-flight mass spectrometry as a tool to distinguish the lot-to-lot heterogeneity in $\mathrm{N}$ glycosylation profile of the therapeutic monoclonal antibody trastuzumab. J. Am. Soc. Mass Spectrom. 2008;20(11):2021-33.

Evans ME, Clark WC, Zheng G, Pan T. Determination of tRNA aminoacylation levels by high-throughput sequencing. Nucleic Acids Res. 2017;45(14):e133.

Ewing MA, Glover MS, Clemmer DE. Hybrid ion mobility and mass spectrometry as a separation tool. J Chromatogr A. 2016;1439:3-25.

Gabelica V, Marklund E. Fundamentals of ion mobility spectrometry. Curr. Opin. Chem. Biol. 2018;42:51-9. https://doi.org/10.1016/j.cbpa.2017.10.022.

Gabelica V, et al. Recommendations for reporting ion mobility mass spectrometry measurements. Mass Spectrom. Rev. 2019;38:291-320.

Giles K, Williams JP, Campuzano I. Enhancements in travelling wave ion mobility resolution. Rapid Commun. Mass Spectrom. 2011;25:1559-66.

Grozhik AV, Jaffrey SR. Distinguishing RNA modifications from noise in epitranscriptome maps. Nat. Chem. Biol. 2018;14(3):215-25.

Ham BM, Maham A. Analytical chemistry: a chemist and laboratory technician's toolkit. Hoboken: Wiley; 2016. p. 559-77.

Hsu PJ, He C. High-resolution mapping of $N^{6}$-methyladenosine using $\mathrm{m}^{6} \mathrm{~A}$ crosslinking immunoprecipitation sequencing ( ${ }^{6} \mathrm{~A}-\mathrm{CLIP}$-Seq). In: Wajapeyee N, Gupta R, editors. Epitranscriptomics. Methods in Molecular Biology, vol. 1870. New York, NY: Humana Press; 2019.

Hsu PJ, et al. Single base resolution mapping of 2'-O-methylation sites in human mRNA and in 3' terminal ends of small RNAs. Methods. 2019;156:85-90.

Huber SM, Leonardi A, Dedon PC, Begley TJ. The versatile roles of the tRNA epitranscriptome during cellular responses to toxic exposures and environmental stress. Toxics. 2019;7(1):17-35.

Kirk AT, et al. Ultra-high-resolution ion mobility spectrometry - current instrumentation, limitations, and future developments. Anal. Bioanal. Chem. 2019;411:6229-46.

Konno $\mathrm{M}$, Taniguchi $\mathrm{M}$, Ishii $\mathrm{H}$. Significant epitranscriptomes in heterogeneous cancer. Cancer Sci. 2019;110(8):2318-27.

Kono N, Arakawa K. Nanopore sequencing: review of potential applications in functional genomics. Dev. Growth Differ. 2019;61(5):316-26.

Kune C, Haler J, Far J, De Pauw E. Effectiveness and limitations of computational chemistry and mass spectrometry in the rational design of target-specific shift reagents for ion mobility spectrometry. ChemPhysChem. 2018;19(21): 2921-30.

Lagies S, et al. Unraveling altered RNA metabolism in pancreatic cancer cells by liquid-chromatography coupling to ion mobility mass spectrometry. Anal Bioanal. Chem. 2019;411(24):6319-28.

Licht K, Jantsch MF. Rapid and dynamic transcriptome regulation by RNA editing and RNA modifications. J. Cell Biol. 2016;213(1):15-22. 
Manasses J, et al. Differentiating positional isomers of nucleoside modifications by high-energy collisional dissociation mass spectrometry (HCD MS). J. Am Soc. Mass Spectrom. 2018;29:1745-56.

May JC, McLean JA. Ion mobility-mass spectrometry: time-dispersive instrumentation. Anal. Chem. 2015;87:1422-36.

Motorin Y, Helm M. Methods for RNA modification mapping using deep sequencing: established and new emerging technologies. Genes. 2019;10(1):E35.

Mwangi JN, Todd DA, Chiu NHL. Evaluating the variation of ion energy under different parameter settings in traveling wave ion mobility mass spectrometry. Int. J. Ion. Mobil. Spec. 2018;21(3):81-6.

Nachtergaele $\mathrm{S}, \mathrm{He}$ C. Chemical modifications in the life of an mRNA transcript. Ann. Rev. Genet. 2018;52:349-72.

Pan T. Modifications and functional genomics of human transfer RNA. Cell Res. 2018;28(4):395-404.

Rose RE, Quinn R, Sayre JL, Fabris D. Profiling ribonucleotide modifications at fulltranscriptome level: a step toward MS-based epitranscriptomocs. RNA. 2015; 21:1361-74.

Shi $\mathrm{H}$, Wei J, He C. Where, when, and how: context-dependent functions of RNA methylation writers, readers, and erasers. Mol. Cell. 2019;74:640-50.

Wetzel C, Limbach P. A. Mass spectrometry of modified RNAs: recent developments. Analyst. 2016;141:16-23.

Zaccara S, Ries RJ, Jaffrey SR. Reading, writing and erasing mRNA methylation Nat. Rev. Mol. Cell. Biol. 2019;20:608-24.

Zhang $L$, et al. Transcriptome-wide mapping of internal n7-methylguanosine methylome in mammalian mRNA. Mol. Cell. 2019a;74(6):1304-16.

Zhang Z, et al. Single-base mapping of m6A by an antibody-independent method. Sci. Adv. 2019b;5(7):eaax0250.

\section{Publisher's Note}

Springer Nature remains neutral with regard to jurisdictional claims in published maps and institutional affiliations.

\section{Submit your manuscript to a SpringerOpen ${ }^{\circ}$ journal and benefit from:}

- Convenient online submission

- Rigorous peer review

- Open access: articles freely available online

High visibility within the field

- Retaining the copyright to your article

Submit your next manuscript at $\boldsymbol{\nabla}$ springeropen.com 